\title{
Multi-element images updated live while the data is collected using a time of flight ICP-MS when all argon and nitrogen interferences are removed with a novel segmented reaction cell interference removal device
}

PHIL SHAW AND LUKAS SCHLATT

$\mathrm{Nu}$ Instruments

Presenting Author: Lukas.Schlatt@ametek.com

Time of Flight (TOF) ICP-MS is developing rapidly with the recent availability of very fast washout laser ablation cells for imaging. The latest laser ablation cells have washout times of only a few milliseconds allowing complete pixel separation of signals whilst ablating at repetition rates of $100-1000 \mathrm{~Hz}$. This makes collecting a small spot-size, large area image a much faster task. However, a lot of information is lost if multiple elements cannot be collected for each image making TOF the optimal mass spectrometer for imaging as full spectra can be collected and stored to disk faster than the laser is ablating and each image pixel can be constructed from simultaneously acquired multi-element data. We will present the ways in which Vitesse is used with the latest laser cells to obtain high density images quickly and easily.

Software will be presented that fully controls the running of patterns on the laser and the acquisition of data with the ICP-MS. The benefits of flexibility in when data is stored and how frequently gas blanks are taken will be shown. The ability to collect multiple images in one automated session will be shown as well as the possibility of inserting calibrations both between and even within the collection of data for each image. The display of fully processed pixel data as the images are acquired on a line by line basis will be shown highlighting the benefit of being able to review whether the sample being measured is valuable data or not so precious analytical time is not wasted.

Data will be shown for a variety of sample types for a broad range of applications and matrices. 